\title{
Ptose palpebral associada a paquidermoperiostose: relato de caso
}

\author{
Eyelid ptosis associated with pachydermoperiostosis:case report
}

\author{
Ana Paula Ximenes Alves ${ }^{1}$ \\ Joana Gurgel Holanda Filha ${ }^{2}$ \\ Fabiane Trindade Jerônimo ${ }^{3}$
}

Trabalho realizado no Departamento de Cirurgia da Faculdade de Medicina da Universidade Federal do Ceará, Setor de Oftalmologia do Hospital Geral de Fortaleza. Ceará (CE).

${ }^{1}$ Doutora em Oftalmologia. Professora substituta da disciplina de Oftalmologia do Departamento de Cirurgia da Faculdade de Medicina da Universidade Federal do Ceará (UFCE). Ceará (CE).

${ }^{2}$ Residente de Oftalmologia, segundo ano, do Serviço de Oftalmologia da UFCE. Ceará (CE).

${ }^{3}$ Residente de Oftalmologia, segundo ano, do Serviço de Oftalmologia do Hospital Geral de Fortaleza. Ceará (CE).

Endereço para correspondência: Rua Tibúrcio Cavalcante, 847 - Apto. 1801 - Fortaleza (CE) CEP 60125-100

E-mail: apxalves@ hotmail.com

Recebido para publicação em 12.05.2004

Versão revisada recebida em 17.11.2004

Aprovação em 17.01.2005

Nota Editorial: Após concluída a análise do artigo sob sigilo edotorial e com a anuência do Dr. Waldir Martins Portellinha sobre a divulgação de seu nome como revisor dele, agradecemos sua participação nesse processo

\begin{tabular}{|c|}
\hline RESUMO \\
\hline Descreve-se caso clínico de um indivíduo acometido por paquidermope- \\
riostose, cuja queixa principal foi ptose palpebral bilateral. Trata-se de \\
doença hereditária, autossômica dominante, que acomete a pele, os ossos \\
e os tecidos moles, resultando em acentuada hipertrofia tarsal. Discute-se \\
a fisiopatologia da ptose e o tratamento executado no caso.
\end{tabular}

Descritores: Blefaroptose/fisiopatologia; Blefaroptose/cirurgia; Osteoartropatia hipertrófica primária/etiologia; Relatos de casos [Tipo de publicação]

\section{INTRODUÇ̃̃̃O}

Paquidermoperiostose ou osteoartropatia hipertrófica primária é uma rara doença hereditária caracterizada por hiperostose, baqueteamento digital e acometimento cutâneo.

Uma das características principais da doença é a paquidermia, espessamento cutâneo causado por hiperplasia endotelial na derme, infiltrado de linfócitos e histiócitos, além de depósito de fibras colágenas ${ }^{(1)}$. Estas alterações costumam afetar a face do paciente, conferindo-lhe feições grosseiras, incluindo as pálpebras.

A hiperostose, por sua vez, consiste na formação de tecido ósseo sob o periósteo do osso normal. Acomete principalmente as extremidades distais dos metacarpos, metatarsos e ossos longos. O baqueteamento digital decorre da proliferação de tecido conectivo no leito ungueal, associada à dilatação e hipertrofia da parede dos pequenos vasos.

Relatamos aqui um caso em que a queixa principal do paciente foi ptose palpebral relacionada à doença.

\section{CASO CLÍNICO}

MAP, sexo masculino, 60 anos, procedente de Camocim, CE, a $360 \mathrm{~km}$ de Fortaleza, agricultor, solteiro, procurou o Hospital Geral César Cals em abril de 2002, com queixa de "problema no olho". Referiu que há cerca de 4 anos vinha evoluindo com dificuldade para abrir os olhos, associada a um aumento da espessura palpebral, pior à direita. Apresentava ainda prurido palpebral e secreção ocular mucóide, crônicos.

Além das queixas oftalmológicas, referia aumento de volume das extremidades desde a infância (pés e mãos) e que vinha evoluindo com aumento de volume e dor nas articulações dos punhos, tornozelos, joelhos, dedos das mãos e ombro (Figura 2). Queixou ainda de cefaléia crônica, adinamia e sudorese profusa. 
Ao exame, apresentava acuidade visual de 20/400 e 20/60 com correção (-9.0 D esf. $x-1.75$ cil $161^{\circ}$ no olho direito e $-2.5 \mathrm{D}$ esf. $x-2.25$ cil $110^{\circ}$ no esquerdo) e exotropia com desvio do olho direito. Apresentava ptose palpebral bilateral severa, que lhe causava dificuldade de locomoção (Figura 1). Mesmo usando os músculos frontais, não era possível visualizar o reflexo corneano (distância margem-reflexo negativa). A fenda palpebral era de 2,0 mm em ambos os olhos; a medida da excursão da pálpebra superior era próxima de zero e não havia sulco palpebral superior. À biomicroscopia evidenciava um aumento importante de espessura dos tarsos e papilas gigantes nos tarsos superiores (Figura 3). Apresentava catarata nuclear mais pronunciada à direita. Não havia alterações corneanas ou na câmara anterior. Tonometria de 20 e $12 \mathrm{mmHg}$ e fundoscopia normal em ambos os olhos.

Foi submetido a uma extensa investigação clínica, que detectou um hipotireoidismo e hipertensão arterial sistêmica. Foram afastadas causas pulmonares, cardíacas, endócrinas ou paraneoplásicas que explicassem seu quadro cutâneo e osteoarticular. O diagnóstico final foi então, de osteoartropatia

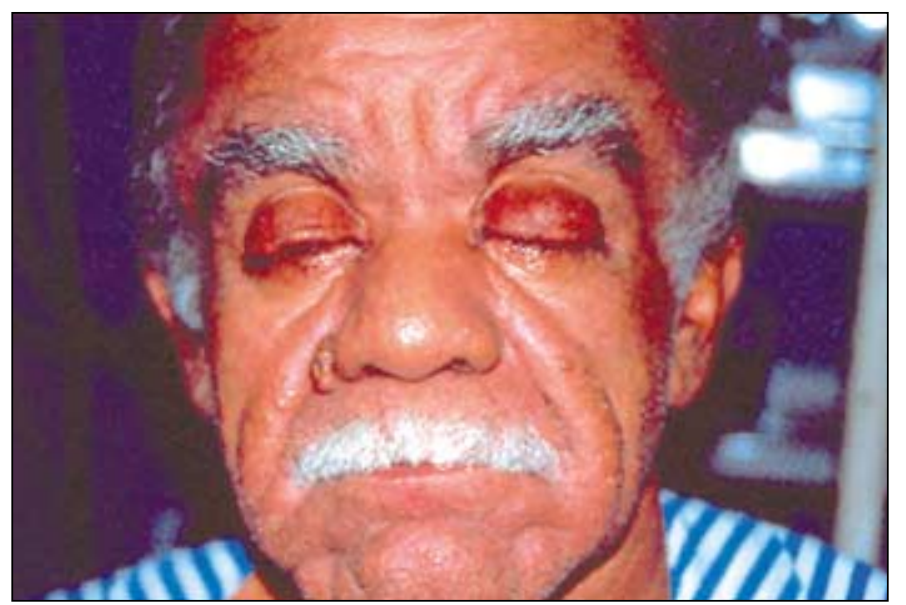

Figura 1 - Ptose palpebral bilateral e incapacitante

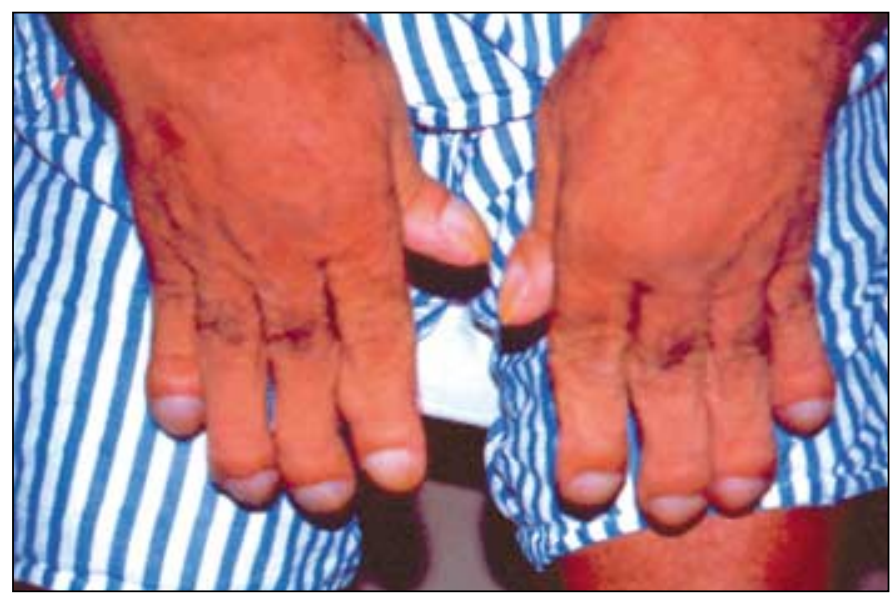

Figura 2 - Aspecto das mãos e baqueteamento digital hipertrófica primária, corroborado pela informação de que um irmão (entre 6) e dois filhos de uma prima do paciente apresentavam alterações sistêmicas semelhantes.

Paralela à investigação clínica, foi submetido a uma biópsia palpebral (exérese de pentágono), que revelou hipertrofia de glândulas sebáceas. Realizou-se, então, um avançamento da aponeurose do elevador, que se encontrava desinserida, sem bom resultado. Num segundo tempo, foi submetido a um encurtamento horizontal das pálpebras superiores e inferiores, conseguindo-se medir uma distância margem-reflexo de 2,0 mm bilateral e satisfação do paciente (Figura 4).

\section{COMENTÁRIOS}

O baqueteamento digital, um dos primeiros sinais clínicos que surgem nas osteoartropatias hipertróficas, foi descrito por Hipócrates em $450 \mathrm{AC}^{(2)}$. Achado semelhante foi visto em esqueletos da América Central, da mesma época ${ }^{(2)}$. Trata-se, portanto, de uma doença reconhecida desde a Antigüidade.

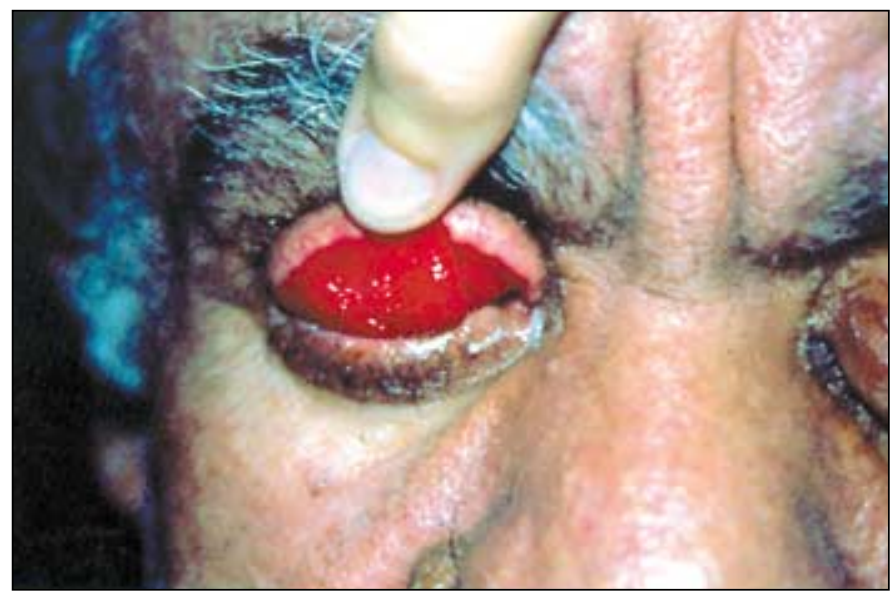

Figura 3 - Hipertrofia tarsal e conjuntival

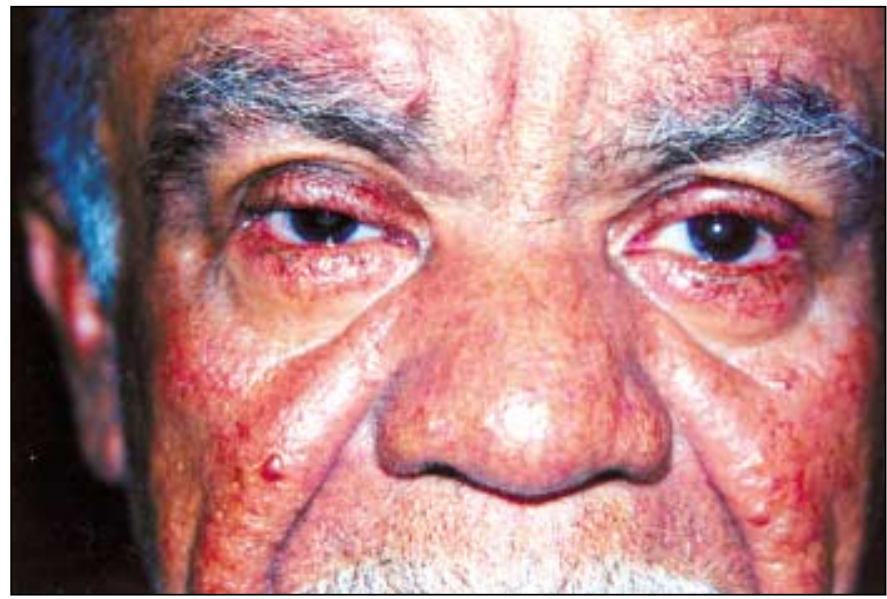

Figura 4 - Aspecto pós-operatório final 
Só mais tarde descobriu-se, porém, duas formas da doença: a osteoartropatia hipertrófica $(\mathrm{OAH})$ primária, também chamada de síndrome de Touraine-Solente-Gole, e a doença secundária, síndrome de Pierre-Marie-Bamberger ${ }^{(3)}$. Esta última é mais prevalente, ocorrendo em associação a doenças pulmonares, cardíacas, hepáticas, intestinais, doença de Graves, entre outras ${ }^{(4)}$. Acredita-se que nestas doenças, fatores de crescimento tecidual normalmente inativos ou removidos na sua passagem pelo pulmão, alcançariam a circulação sistêmica na sua forma ativa, e induziriam às alterações vistas na osteoartropatia hipertrófica. Distúrbios de plaquetas, fibroblastos e células endoteliais foram descritos ${ }^{(5)}$.

A OAH primária consiste em doença hereditária, autossômica dominante, mais comum em homens ${ }^{(6)} \mathrm{e} \mathrm{de} \mathrm{fisiopatologia}$ ainda desconhecida. Estudos apontam para distúrbio primário dos fibroblastos, envolvendo proliferação anormal ${ }^{(7)} \mathrm{e}$ síntese aumentada de glicosaminoglicanas ${ }^{(8)}$. Tem início na infância, progride por alguns anos e estaciona. Na pele, além da paquidermia, verificam-se alterações glandulares, como hiperhidrose, hipertrofia sebácea, acne e foliculite.

As pálpebras encontram-se espessadas, sobretudo o tarso, às custas de hipertrofia das glândulas de Meibomius ${ }^{(9-10)}$, o que é facilmente explicado pela hipertrofia sebácea generalizada vista na doença. Este espessamento acaba por produzir ptose mecânica, associada ou não a desinserção da aponeurose do elevador $^{(10-11)}$. No caso descrito, o componente mecânico foi mais importante na fisiopatologia da ptose, tendo em vista que o melhor resultado cirúrgico só foi conseguido após o encurtamento das pálpebras. Os autores avaliam que o encurtamento horizontal foi benéfico nesse tipo de paciente porque diminuiu a massa palpebral e assim, o efeito mecânico da hipertrofia tarsal. Raciocínio semelhante explica porque uma tarsectomia transconjuntival também pode dar bom resultado ${ }^{(11)}$.

Vale ressaltar que as pálpebras inferiores também foram encurtadas porque obstruíam o eixo visual do paciente no olhar para baixo. Quadro semelhante é visto em alguns casos de retração de pálpebra inferior que recebem enxerto ou implante espaçador e evoluem com distúrbio da motilidade palpebral $^{(12)}$. No nosso paciente isso ocorre em decorrência da grande hipertrofia tarsal e conseqüente falência dos retratores em movimentá-lo.

É interessante observar que o paciente desconhecia a sua doença sistêmica e aceitou-a até que a ptose palpebral comprometesse as suas tarefas diárias, quando veio à Fortaleza procurar tratamento. Tal grau de acometimento oftalmológico na paquidermoperiostose não parece ser comum, visto que, na literatura nacional, os relatos de caso privilegiam os aspectos ósseos e cutâneos da doença ${ }^{(13-21)}$.

Outro aspecto curioso do caso é que o paciente relata que os seus parentes acometidos pela OAH também já têm comprometimento palpebral significativo, mas não se dispõem a procurar assistência médica especializada. Isto reflete a dificuldade de acesso ao oftalmologista, que se concentra nas grandes cidades e não atende à população rural e dos pequenos centros urbanos (Camocim tem cerca de 51000 habitantes e fica a $360 \mathrm{~km}$ de Fortaleza).
A biópsia palpebral mostrou alterações inespecíficas, compatíveis com a doença, mas incapazes de elucidar o caso. Só a investigação clínica minuciosa, que afastou diagnósticos diferenciais como acromegalia, insuficiência cardíaca e pulmonar, foi capaz de chegar ao diagnóstico correto.

Além da ptose, estes pacientes podem apresentar distrofia macular, uma distrofia corneana da membrana de Bowman, e leucoma corneano ${ }^{(22)}$. O tratamento sistêmico é feito com antiinflamatórios não hormonais, para alívio da dor. Na falência desses, pode-se fazer uso de bisfosfonados ${ }^{(23)}$.

\section{AGRADECIMENTOS}

Ao setor de Clínica Médica do Hospital César Cals de Fortaleza, ao setor de Oftalmologia do Hospital Geral e do Hospital Universitário de Fortaleza.

\section{ABSTRACT}

We describe a clinical case of a patient with pachydermoperiostosis and bilateral palpebral ptosis. It is a hereditary, dominant autosomic disease, which affects skin, bones and soft tissues. In the eyelid, it causes an important tarsal hypertrophy. We discuss the physiopathology and treatment of the ptosis.

Keywords: Blepharoptosis/physiopathology; Blepharoptosis/surgery; Osteoarthropathy, primary hypertrophic/etiology; Case reports [Publication type]

\section{REFERÊNCIAS}

1. Matucci-Cerinic M, Lotti T, Calvieri S, Ghersetich I, Sacerdoti L, Teofoli P, et al. The spectrum of dermatological symptoms of pachydermoperiostosis (primary hypertrophic osteoarthropathy): a genetic, cytogenetic and ultrastructural study. Clin Exp Rheumatol. 1992;10 Suppl 7:45-8.

2. Martinez-Lavin M, Mansilla J, Pineda C, Pijoan C, Ochoa P. Evidence of hypertrophic osteoarthropathy in human skeletal remains from pre-Hispanic Mesoamerica. Ann Intern Med. 1994;120(3):238-41.

3. Eckardt A, Kreitner KF. [Primary hypertrophic osteoarthropathy]. Z Orthop Ihre Grenzgeb. 1995;133(4):303-5. German.

4. Lavín-Martinez M. Regional and heritable bone and collagen diseases/hypertrophic osteoarthropathy. In: Klippel JH, Dieppe PA. Rheumatology. 2nd ed. London: Mosby; 1998. p.46.1-46.4.

5. Silveira LH, Martinez-Lavín M, Pineda C, Fonseca MC, Navarro C, Nava A. Vascular endothelial growth factor and hypertrophic osteoarthropathy. Clin Exp Rheumatol. 2000;18(1):57-62.

6. Jansen T, Brandl G, Bandmann M, Meurer M. [Pachydermoperiostosis]. Hautarzt. 1995;46(6):429-35. German.

7. Matucci-Cerinic M, Sacerdoti L, Perrone C, Carossino A, Cagnoni ML, Jajic I, Lotti T. Pachydermoperiostosis (primary hypertrophic osteoarthropathy): in vitro evidence for abnormal fibroblast proliferation. Clin Exp Rheumatol. 1992;10 Suppl 7:57-60.

8. Wegrowski Y, Gillery P, Serpier H, Georges N, Combemale P, Kalis B, Maquart FX. Alteration of matrix macromolecule synthesis by fibroblasts from a patient with pachydermoperiostosis. J Invest Dermatol. 1996; 106(1):70-4.

9. Kirkpatrick JN, McKee PH, Spalton DJ. Ptosis caused by pachydermoperiostosis. Br J Ophthalmol. 1991;75(7):442-6.

10. Friedhofer H, Salles AG, Gemperli R, Ferreira MC. Correction of eyelid anomalies in pachydermoperiostosis. Ophthal Plast Reconstr Surg. 1999; 15(2):137-8. 
11. Arinci A, Tumerdem B, Karan MA, Erten N, BuyukbabanI N. Ptosis caused by pachydermoperiostosis. Ann Plast Surg. 2002;49(3):322-5.

12. Wearne MJ, Sandy C, Rose GE, Pitts J, Collin JR. Autogenous hard palate mucosa: the ideal lower eyelid spacer? Br J Ophthalmol. 2001;85(10):1183-7.

13. Carvalho TN, Araújo Júnior CR, Fraguas Filho SR, Costa MAB, Teixeira KISS, Ximenes CA. Osteoartropatia hipertrófica primária (paquidermoperiostose): relato de casos em dois irmãos. Radiol Bras. 2004;37(2):147-9.

14. Batista AAP, Bianco JAP, Batista AP, Alves LR, Afonso A, Silva HSL, et al. Osteoartropatia hipertrófica primária: relato de caso e revisão da literatura. Radiol Bras. 2003;36(3):183-6.

15. Ribeiro RC, Santos OLR, Moreira A, Oliveira Neto MP. Paquidermoperiostose. J Bras Med. 1993;64(6):43-6,50,52.

16. Cruz MA, Pereira GJC, Pereira HR. Osteoartropatia hipertrófica primária: descrição de um caso e revisão da literatura. J Bras Med. 1995;69(5/6):145-62.

17. Macedo LMG, Tormin FB, Gonçalves HAT, Nóbrega MB, Ferreira MS Maia ABA, et al. Paquidermoperiostose: relato de um caso e revisão da literatura. Rev Bras Reumatol. 1990;30(2):67-70.
18. Inocêncio RM, Moreira HNC, Bertolo MB, Samara AM. Osteoartropatia hipertrófica primária (paquidermoperiostose): relato de dois casos e revisão da literatura. Rev Bras Reumatol. 1992;32(1):34-40.

19. Marques GC, Seragini FC, Kuester IMJ, Maio E. Paquidermoperiostose. Klinikos. 1987;3(12):17-9.

20. Laredo Filho J, Lazzareschi M, Braga Júnior MB, Kasinski S, Brizzi JJ. Paquidermoperiostose: atrofia hipertrófica primária: estudo de dois pacientes. Rev Imagem. 1986;8(1):1-8.

21. Souza BDB, Beltreschi F, Russo Filho FS, Carlquist I. Paquidermoperiostose: relato de caso. Rev Bras Reumatol. 1985;25(6):209-10.

22. Orentreich DS, Orentreich N. Dermatology of the eyelids (excluding neoplasms). In: Nesi FA, Levine MR, Lisman RD. Smith's ophthalmic plastic and reconstructive surgery. 2nd ed. St. Louis: Mosby; 1997. p.515-6.

23. Guyot-Drouot MH, Solau-Gervais E, Cortet B, Deprez X, Chastanet P, Cotten A, Delcambre B, Flipo RM. Rheumatologic manisfestations of pachydermoperiostosis and preliminary experience with bisphosphonates. J Rheumatol. 2000;27(10):2418-23. 